\title{
Structural studies on the GKCR7 decoy receptor
}

\author{
T. De Vero', S. Costantini ${ }^{2 \bowtie}$, G. Colonna ${ }^{3}$ \\ 'Dipartimento di Farmacologia Sperimentale, Università degli Studi di Napoli "Federico II", Italy \\ ${ }^{2}$ INT Pascale - Centro Ricerche Oncologiche Mercogliano, Mercogliano, Italy \\ ${ }^{3}$ Dipartimento di Biochimica e Biofisica '\& Centro di Ricerca Interdipartimentale di Scienze Computazionali e \\ Biotecnologiche, Seconda Università di Napoli, Italy
}

\section{Motivations}

Recent studies have revealed that the chemokine receptor CXCR7 plays an important role in cancer development. However, little is known about the effect of CXCR7 on the process of hepatocellular carcinoma $(\mathrm{HCC})$ cell invasion and angiogenesis. In particular, CXCR7 was overexpressed in HCC tissues. The CXCR7-receptor is a transmembrane protein that binds two chemokines, CXCL11 and CXCL12. It has the same basic structure of the other chemokine receptors composed by 7 helices and some Cys residues within the primary sequence to stabilize the tertiary structure through intermolecular disulfide bonds. The signal transduction of chemokines occurs, usually via the $G$ proteins whereas CXCR7 is a "decoy receptor" and behaves as the "receptor Duffy". In particular, it does not bind to $G$ protein, and then does not activate its metabolic pathway. When CXCR7 binds to CXCL12, it can form homodimers or heterodimers from the moment that tends to bind also to CXCR4. In the case of heterodimer (CXCL12/CXCR4/CXCR7), CXCR7 changes the conformation of the CXCR4/ $G$-protein complex and repealing signaling. The activation of CXCR4 by CXCL12 leads to the activation of signaling through PI3K/AKT, IP3, and MAPK pathways, that promote cell survival, proliferation and chemotaxis. In addition, the pathway of the $\beta$-arrestin may be activated by GRK that leads to the internalisation of CXCR4. When CXCR7 alloy CXCL12, the mobilization of intracellular Ca2 + classical does not occur, and the activation of $\beta$-arrestin may lead to the scavenging.CXCL12. In tumor cells CXCR7 can also activate the signaling via PLC / MAPK that increases the survival of the cells. Therefore, to study the structure-function relationships in CXCR7, we have modeled its three dimensional structure as well as studied the energetic stability of the protein by molecular dynamics simulations. Finally, its complex with CXCL11 and CXCL12 has been simulated by docking methods.

\section{Methods}

The three-dimensional model of human CXCR7 was performed by a comparative modeling strategy using as template the structure of the human CXCR4, recently published, and that of bovine rhodopsin using MODELLER9v5 program. Models have been evaluated in terms of stereochemical, structural packing and energetic quality. PatchDock web server was used to model the complexes between CXCR7 and the two ligands, CXCL11 and CXCL12, using for CXCR7 our model obtained by comparative modeling, for CXCL11 and CXCL12 their experimental structures. The complexes were analyzed by "Cocomaps Server" to identify the amino acids at the interface and to evaluate their solvent accessibility. The presence of putative $\mathrm{H}$-bonds was calculated with Hbplus program.

\section{Results}

Our studies show that CXCR7 model presents seven trans-membrane helices, a long N-terminal segment and three loops (i.e. loops 1, 2 and 3) in the extracellular region and, moreover, other three loops and a C-terminal loop in the cytoplasmatic region can also be observed. This model had a Prosa Z-score of -2.19 and $86.54 \%$ of residues in most favored regions. In particular, the extracellular loop 2 and the cytoplasmatic C-terminal region comprise two short $\beta$-strands and a short helix, respectively, in agreement with the experimental structure of CXCR4. A comparison between the secondary structures of CXCR7 and CXCR4 models shows seven well conserved trans-membrane helices and only few changes in helix length. Furthermore, we have modeled two complexes between CXCR7 and two chemokines, CXCL11 and CXCL12. For these complexes, we evaluated the interaction residues, the related contact maps and the number of inter-chain H-bonds. In general, the analysis of the two complexes shows that the interacting regions are located in the $\mathrm{N}$-terminal region as well as in the loops 2 and 3 of CXCR7. We have 
also analyzed the physico-chemical properties of interaction residues of CXCR7 in the two complexes: i) some positively and negatively charged residues are present in the $\mathrm{N}$-terminal while in the CXCR7/CXCL11 complex we also found also an aromatic residue; ii) the loop 2 presents a negatively charged residue (Glu) and an aromatic residue (Tyr); iii) the loop 3 shows a single aromatic residue (Phe). These observations suggest that the predominant interactions found in the models between CXCR7 and the two chemokines are on hydrophobic and electrostatic basis. In particular, CXCR7 presents the highest affinity for CXCL12 in terms of binding energy, and of number of $\mathrm{H}$-bonds, of charged and hydrophobic interaction residues, and of salt bridges. Moreover, the presence of stacking interactions is also in good agreement with experimental studies which indicated that the affinity of CXCL12 for CXCR7 is approximately ten times higher than the affinity of CXCL12 for CXCR4. 